INVITED COMMENTARY

\title{
Incidentaloma, glucocorticoid excess and low bone mineral density: a coincidence?
}

\author{
Jean-Pierre Devogelaer \\ Rheumatology Unit, St-Luc University Hospital, Université catholique de Louvain, avenue Hippocrate 10, B-1200 Brussels, Belgium \\ (Correspondence should be addressed to Jean-Pierre Devogelaer; Email: devogelaer@ruma.ucl.ac.be)
}

The paper by L Tauchmanovà et al. published in this issue of the Journal leads to the discussion of several aspects of the management of patients suspected of being exposed to glucocorticoid excess (1). The first point to be considered is that there is no doubt, nowadays, that glucocorticoid (GC) excess, whether exogenous or endogenous, leads to rapid bone loss early after the start of GC therapy and, by extrapolation, presumably early in the course of excessive adrenal secretion. It increases dramatically the risk of fragility fractures (2). Osteoporosis (OP) had already been observed by $\mathrm{H}$ Cushing in his princeps description of the syndrome which, since then, bears his name. The mechanisms of glucocorticoid-induced osteoporosis (GC-OP) are multiple, but not yet fully understood. Grossly, GC-OP is the consequence of decreased bone formation and increased bone resorption, the former being more marked than the latter (3). Both act synergistically to provoke bone fragility. Increased bone resorption may be the consequence of some degree of secondary hyperparathyroidism (4), only partially explained by a GC-induced sensitization to parathyroid hormone (PTH) action of the target cells. Secondary hyperparathyroidism mainly results from the decrease in intestinal calcium absorption, probably induced by some acquired resistance to $1,25(\mathrm{OH})_{2}$ vitamin $\mathrm{D}_{3}$, and, to a lesser extent, by an increase in urinary calcium excretion, both concurring additively to a trend leading to the lowering of serum calcium. Moreover, GCs inhibit osteoblastic function, as can be seen in dynamic histomorphometry (4) and which is patently illustrated by the dramatic decrease in the plasma osteocalcin level, a marker of osteoblastic function, immediately after GC exposure, with fast recovery in the hours to days following complete withdrawal of GCs (5). Another mechanism for the development of GC-OP could be an increased apoptosis of osteoblasts and osteocytes, both cells so crucial for bone formation and maintenance of bone integrity (6). So far, most of the clinical studies dealing with the bone effect of GCs have been conducted in patients with exogenous GC excess given as therapy for disorders like rheumatoid arthritis and systemic lupus erythematosus, which may themselves affect bone turnover and bone mineral density (BMD). In Cushing's syndrome, however, similar alterations of bone turnover and BMD have been observed (7). The complexity of the mechanisms potentially leading to GC-OP can help to understand why, at least in some patients, even very low doses of endogenous or exogenous GCs might be deleterious to bone and lead to an increased risk of vertebral and non-vertebral fractures (8). Histomorphometrically, with doses up to $10 \mathrm{mg}$ there is a thinning of bone trabeculae with long-term maintenance of interconnectivity $(9-11)$, as opposed to postmenopausal OP in which perforations of trabeculae occur rapidly, leading to a rapid degradation of bone microarchitecture. With higher GC doses, however, irreversible trabecular discontinuity is observed (10, 11). If extrapolated to what happens in incipient Cushing's syndrome, it seems, therefore, mandatory to diagnose as early as possible any excessive endogenous secretion of GC, in order to prevent a dramatic bone loss and the destruction of bone architecture. This is an easy task if the results of testing the hypothalamicpituitary-adrenal axis are clearcut. Indeed, several reports have observed recovery of bone mineral density after withdrawal of GCs or after curative therapy of overt Cushing's syndrome (12). This should encourage practitioners to check systematically the bone mineral density not only in patients chronically on GC therapy (any dosage) but also in patients in whom endogenous glucocorticoid excess is suspected.

The second point is how to measure BMD. It looks a more ancillary issue, as far as the choice of the technique is concerned, if only a cross-sectional (as opposed to longitudinal) BMD measurement is envisaged. Currently, dual energy X-ray absorptiometry (DXA) is the more widely accepted procedure, well validated for longitudinal trials. The risk-definitions for postmenopausal osteoporosis with their expression as T-scores, proposed by a World Health Organisation (WHO) group, are based on this technique (13). However, these definitions are only validated for Caucasian postmenopausal women, and are not directly applicable to males nor to secondary osteoporosis. Ultrasound techniques have also been developed. They are able to measure various sites of the skeleton like calcaneus, tibia, and the phalanges of the fingers. Some further developments are expected. However, each manufacturer develops his own apparatus with its proper characteristics, potentially leading 
to some confusion among users. Their attractive value is that they do not use ionizing radiation, and are cheaper and more portable than conventional bone densitometers using DXA that scan the spine and the femur, two sites central to fragility fractures. Whether peripheral ultrasonography can predict fracture risk as accurately as DXA has been suggested in studies on senile and postmenopausal osteoporosis, at least for the measurement of the heel in a water bath (14). So far, it has not been demonstrated that this will also be the case in GC-OP for both calcaneus and finger ultrasonography, the latter elegant technique of measurement having been used by L Tauchmanovà et al. (1). According to their results, ultrasonography might at least be useful to preselect patients in whom more cumbersome axial and femoral DXA is indicated to confirm/exclude osteoporosis at the spine or hip, when there remains some doubt about a correct diagnosis.

A third point is to determine whether patients with adrenal incidentaloma (AI) are at significant increased risk of osteoporosis. Since the introduction in clinical practice of performant diagnostic tools such as ultrasound, computed tomography (CT scan) and magnetic resonance imaging (MRI), AI is more and more frequently observed. Quite a few studies have approached this question, but they seemingly yield different responses. Only small cross-sectional studies are currently available. Torlontano et al. (15) studied BMD in 32 female patients suffering from AI by two different techniques: by single energy quantitative computed tomography (L1-L4) (QCT), a technique which is able to measure pure trabecular BMD, and by DXA of the spine (L2-L4) and of the hip. A significant bone loss was observed in the small subgroup with subclinical hypercortisolism only, as compared with values of the reference population at their center. Their 64 controls tested were in the normal range. In another study, Rossi et al. (16) from the same group as Tauchmanovà (1) evaluated BMD in 18 patients by DXA of the lumbar spine in patients under 65 years ( $n=$ not given) and of the right femoral neck in those older than 65 years $(n=$ not given). They were compared with the international pooled sample provided as reference value by the densitometer's manufacturer. No local control group was available. The BMD data looked consistent with the lack of significant bone loss in patients with adrenal adenoma, even when associated with subclinical Cushing's syndrome. More recently, Osella et al. (17) have measured BMD also by DXA at the lumbar spine and at the hip in 27 patients with AI, with reference to the manufacturer's normative data. A local control group $(n=54)$ had their lumbar spine measured, but unfortunately not the hip because of 'budgetary restrictions'. Lumbar BMD values in patients with $\mathrm{AI}$ were not significantly different from those in control subjects which, however, had BMD values a little lower (but not significantly) than the manufacturer's reference group. Nevertheless, the ranges of the Z-scores (numbers of standard deviations from the mean BMD of the age- and sex-matched manufacturer's controls) were quite large: -3.86 to +1.79 versus -2.96 to +2.67 S.D. in the studied group and the control group respectively, underlying the large variation of BMD values in the studied population. Forty-eight percent of the patients were in the osteopenia and osteoporosis ranges according to the WHO rules (13). The authors also observed a good correlation between BMD values and serum PTH levels, and, as mentioned above, secondary hyperparathyroidism has been implicated in the pathogenesis of GC-OP. In the present study (1), 34 patients were studied both by DXA at the lumbar spine and at the hip, and by ultrasonography (US) of the proximal phalanges; reference values were those provided by the manufacturers. All patients had significantly lower BMD and US values as compared with controls. Bone loss was consistently more marked in patients with overt Cushing's syndrome as compared with patients with subclinical Cushing's syndrome. How can the apparent discrepancies between studies performed in the same country and even in the same center be explained? First, the numbers of studied patients are quite low. Secondly, the criteria to define subclinical Cushing's syndrome may differ from one study to the other. They were less stringent in one study (15), but look similar in others $(1,16,17)$. Thirdly, the techniques of BMD measurements also differed, from ultrasonography of the phalanges, DXA of the lumbar spine and/or the hip, to QCT of the spine, and by comparison to local controls or to the manufacturers' reference values.

What approach should the clinician adopt in the near future when dealing with a patient suffering from AI? The first step should be to rule out the hypothesis of a primary or a secondary metastatic lesion. If the tumor proves to be benign, how does one determine which small tumor will deserve a surgical removal? Besides the lack of knowledge of the natural history of patients with an apparently silent AI, there remains some uncertainty about the long-term safety of subclinical Cushing's syndrome. In the presence of excessive glucocorticoid secretion, the search for any complication like hypertension, diabetes, obesity and dyslipidemia seems advisable. BMD and bone metabolism assessment should also be reasonably added to the decision tree for a therapeutic intervention. A low BMD should help to decide in favor of a surgical operation, somewhat in a similar manner that low BMD is thought to have a role in the indication for parathyroid surgery in asymptomatic hyperparathyroidism (18). Long-term longitudinal studies of bone metabolism and BMD in patients with asymptomatic AI are urgently needed to help to understand better the clinical implication of subclinical Cushing's syndrome in a complicated pathological issue such as GC-OP. 


\section{References}

1 Tauchmanovà L, Rossi R, Nuzzo V, del Puente A, Esposito-del Puente A, Pizzi C et al. Bone loss determined by quantitative ultrasonometry correlates inversely with disease activity in patients with endogenous glucocorticoid excess due to adrenal mass. European Journal of Endocrinology 2001145 241-247.

2 Adinoff AD \& Hollister JR. Steroid-induced fractures and bone loss in patients with asthma. New England Journal of Medicine $1983309265-268$.

3 Reid IR. Pathogenesis and treatment of steroid osteoporosis. Clinical Endocrinology 198930 83-103.

4 Bressot C, Meunier PJ, Chapuy MC, Lejeune E, Edouard C \& Darby AJ. Histomorphometric profile, pathophysiology and reversibility of corticosteroid-induced osteoporosis. Metabolic Bone Disease and Related Research 19791 303-311.

5 Godschalk MF \& Downs RW. Effects of short-term glucocorticoids on serum osteocalcin in healthy young men. Journal of Bone and Mineral Research 19883 113-115.

6 Weinstein RS \& Manolagas SC. Apoptosis and osteoporosis. American Journal of Medicine $2000 \mathbf{1 0 8} 153-164$.

7 Chiodini I, Carnevale V, Torlontano M, Fusilli S, Guglielmi G, Pileri $\mathrm{M}$ et al. Alterations of bone turnover and bone mass at different skeletal sites due to pure glucocorticoid excess: study in eumenorrheic patients with Cushing's syndrome. Journal of Clinical Endocrinology and Metabolism 199883 1863-1867.

8 Vanstaa TP, Leufkens HGM, Abenhaim L, Zhang B \& Cooper C. Use of oral corticosteroids and risk of fractures. Journal of Bone and Mineral Research 200015 993-1000.

9 Aaron JE, Francis RM, Peacock M \& Makins NB. Contrasting microanatomy of idiopathic and corticosteroid-induced osteoporosis. Clinical Orthopaedics and Related Research 1989243 294-305.

10 Chappard D, Legrand E, Basle MF, Fromont P, Racineux JL, Rebel A et al. Altered trabecular architecture induced by corticosteroids: a bone histomorphometric study. Journal of Bone and Mineral Research 199611 676-685.

11 Carbonare LD, Arlot ME, Chavassieux PM, Roux JP, Portero NR \& Meunier PJ. Comparison of trabecular bone microarchitecture and remodeling in glucocorticoid-induced and postmenopausal osteoporosis. Journal of Bone and Mineral Research 200116 97-103.

12 Manning PJ, Evans MC \& Reid IR. Normal bone and mineral density following cure of Cushing's syndrome. Clinical Endocrinology $1992 \quad 36 \quad 229-234$.

13 Kanis JA, Devogelaer JP \& Gennari C. Practical guide for the use of bone and mineral measurements in the assessment of treatment of osteoporosis: a position paper of the European Foundation for Osteoporosis and Bone Disease. Osteoporosis International $19966256-261$.

14 Hans D, Dargent-Molina P, Schott AM, Sebert JL, Cormier C, Kotzki PO et al. Ultrasonographic heel measurements to predict hip fracture in elderly women: the EPIDOS prospective study. Lancet 1996348 511-514.

15 Torlontano M. Chiodini I, Pileri M. Guglielmi G, Cammisa M, Modoni $\mathrm{S}$ et al. Altered bone mass and turnover in female patients with adrenal incidentaloma: the effect of subclinical hypercortisolism. Journal of Clinical Endocrinology and Metabolism $1999842381-2385$.

16 Rossi R, Tauchmanovà L, Luciano $\mathrm{A}$, Di Martino $\mathrm{M}$, Battista $\mathrm{C}$, Del Viscovo L et al. Subclinical Cushing's syndrome in patients with adrenal incidentaloma: clinical and biochemical features. Journal of Clinical Endocrinology and Metabolism $2000 \mathbf{8 5}$ 1440-1448.

17 Osella G, Reimondo G, Peretti P, Ali A, Paccotti P, Angeli A et al. The patients with incidentally discovered adrenal adenoma (incidentaloma) are not at increased risk of osteoporosis. Journal of Clinical Endocrinology and Metabolism 200186 604-607.

18 Silverberg SJ, Locker FG \& Bilezikian JP. Vertebral osteopenia: a new indication for surgery in primary hyperparathyroidism. Journal of Clinical Endocrinology and Metabolism $1996 \mathbf{8 1}$ 4007-4012.

Received 12 April 2001

Accepted 25 May 2001 